\title{
Pellagra affecting a patient with Crohn's disease*
}

\author{
Shi Hui ${ }^{1}$ \\ Wang Shaodong ${ }^{1}$ \\ Wang Zhenkai ${ }^{1}$
}

\author{
Lu Heng ${ }^{1}$ \\ Wang Fangyu ${ }^{1}$
}

DOI: http://dx.doi.org/10.1590/abd1806-4841.20174461

\begin{abstract}
Pellagra is a nutritional disease caused by a deficiency of niacin. It may lead to death if not identified and treated timely. We review the literature and report a female patient presented with clinical features of pellagra as a complication of Crohn's disease.
\end{abstract}

Keywords: Crohn's disease; Niacin; Niacinamide; Pellagra

Pellagra was first described in 1735 by the Spanish physician Gaspar Casal. Chronic alcoholism can be a main cause of pellagra in some developed countries. ${ }^{1}$ In developing countries, the disease may be increasing among HIV-positive patients. Other causes include carcinoid syndrome, Hartnup disease, infection, and isoniazid or fluorouracil therapy. We report here a case of pellagra affecting a Crohn's disease (CD) patient, which is rarely reported in China.

A 49-year-old woman with CD was admitted to our hospital with abdominal pain, diarrhea and a temperature of $38^{\circ} \mathrm{C}$ in 2014 . She had been diagnosed with CD for three years and was treated with mesalazine and prednisone. In 2012, a lower enteroscopy showed fistulas arising from the transverse colon, ileum and descending duodenum. In April 2013, the disease flared for the second time and infliximab was administered for 3 times with unsatisfactory results. After that, the patient followed a very strict diet that was basically limited to pasta, avoiding red meat, fish, milk, and eggs while continuing the treatment with mesalazine.

On admission, the patient had normal weight and normal blood pressure with temperature of $37.6^{\circ} \mathrm{C}$. Physical examination only revealed the surgery scars and skin change without itches or pain. Dark-brown coloration and scaly desquamation of the skin were noticed on the neck, dorsum of the hands, and ankles, which would aggravate after sun exposure (Figure 1). The neck and ankles were tender.
Laboratory test results at admission were as follows: hemoglobin $62 \mathrm{~g} / \mathrm{L}$ with microcytic indices; $5.2 \times 10^{9} / \mathrm{L}$ leukocytes and $228 \times 10^{9}$ /L platelets; CRP $143.1 \mathrm{mmol} / \mathrm{L}$; ESR $95 \mathrm{~mm} / \mathrm{h}$; albumin $27.0 \mathrm{~g} / \mathrm{L} ; \mathrm{BUN} 1.9 \mathrm{mmol} / \mathrm{L} ; \mathrm{SCr} 40 \mu \mathrm{mol} / \mathrm{L}$; iron $2.5 \mu \mathrm{mol} / \mathrm{L}$; and calcium $1.9 \mathrm{mmol} / \mathrm{L}$. Stool routine examination revealed $\mathrm{OB}+$ with no parasites or protozoa. Cultures for Shigella, Campylobacter, and Yersinia species were negative.

A biopsy on the dorsum of the left hand was performed with permission of the patient. Histological examination revealed parakeratotic hyperkeratosis together with a somewhat atrophic epidermis characterized by mild spongiosis, pallor, and ballooning. Dilated vessels with extravasation of red blood cells could be observed in the dermis (hematoxylin-eosin staining, original magnification (Figures 2 and 3).

The patient was then treated with intravenous multi vitamin B concentrate, which included nicotinamide $(50 \mathrm{mg} / \mathrm{d}$ for 5 days) and nicotinamide tablets $(150 \mathrm{mg} / \mathrm{d}$ for 5 days and $300 \mathrm{mg} / \mathrm{d}$ for the following 4 weeks). We placed a gastroscopy-guided nasojejunal feeding tube at the Treitz ligament to provide enteral nutrition, which included nicotinic acid $18 \mathrm{mg} / \mathrm{d}$ with controlled diets. H\&E staining revealed some improvement 10 days after treatment (Figure 4).

Pellagra is rarely reported as a complication of CD caused by a deficiency of niacin. ${ }^{2}$ The prevalence of the disease might be misleading due to underdiagnosis. ${ }^{3}$ Pellagra is divided into two forms:

Received on 14.02.2015.

Approved by the Advisory Board and accepted for publication on 10.06.2016.

* Work performed at the Department of Gastroenterology and Hepatology, Jinling Hospital affiliated to Southern Medical University - Guangdong, China Financial support: none.

Conflict of interest: none.

1 Department of Gastroenterology and Hepatology, Jinling Hospital, China.

C2017 by Anais Brasileiros de Dermatologia 


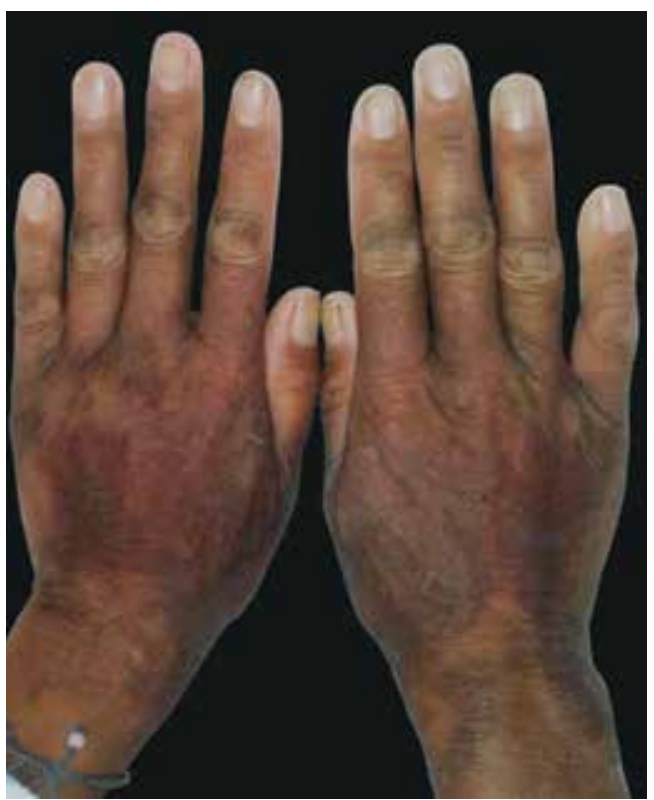

FiguRE 1: Before treatment

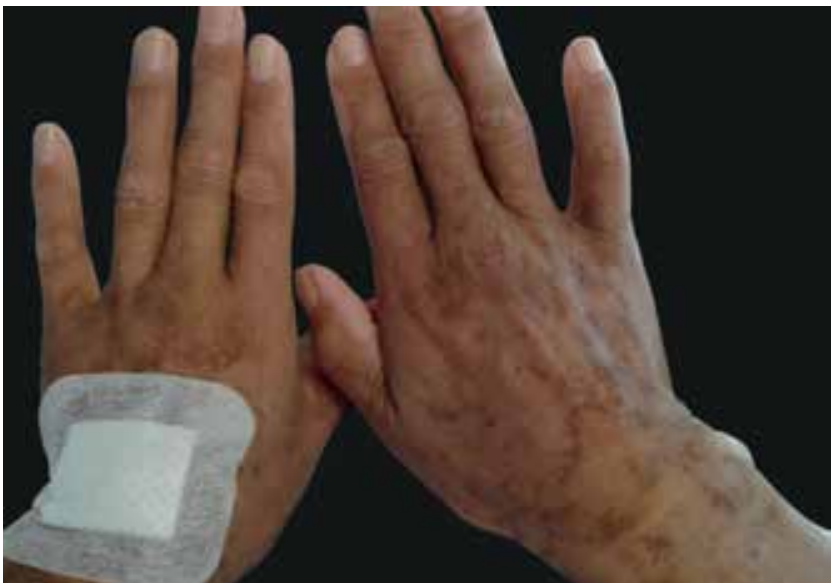

Figure 2: After treatment

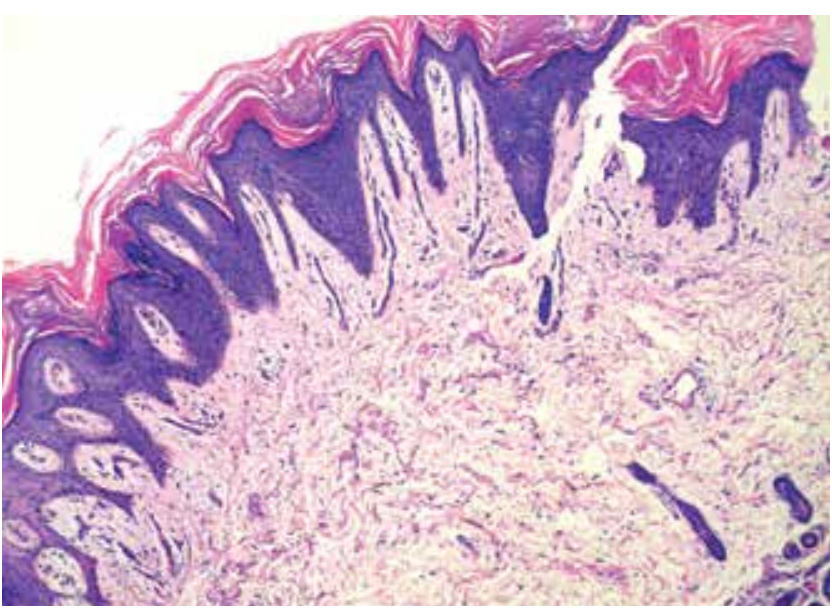

FigURE 3: Pathology of the skin change (Hematoxylin \& eosin X100)

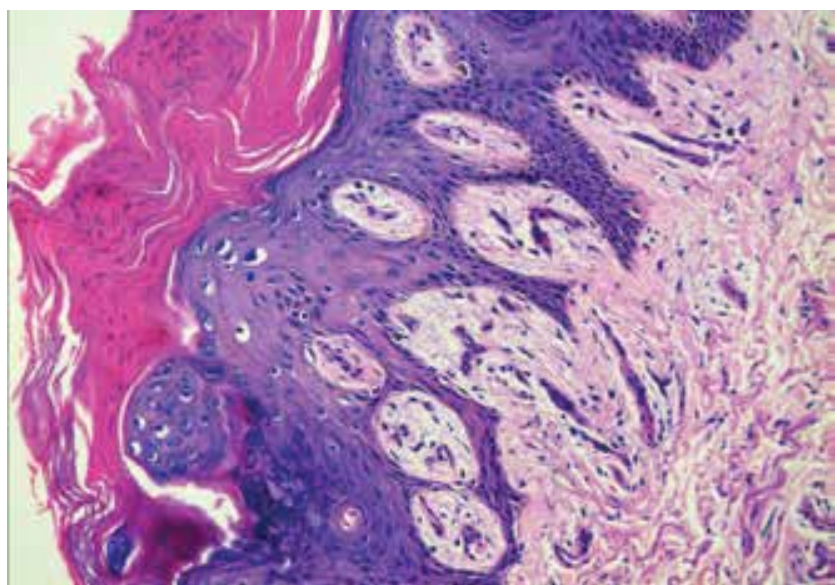

Figure 4: Pathology of the skin change( Hematoxylin \& eosin X200)

primary pellagra, which results from inadequate niacin intake; and secondary pellagra, which occurs when other conditions interfere with niacin absorption and/or processing. These include chronic alcoholism, prolonged diarrhea, and biochemical impairment of tryptophan metabolism (which can be caused by diseases such as carcinoid syndrome, Crohn's disease, and Hartnup disease). ${ }^{4}$ Some drugs - such as levodopa, isoniazid, 6-mercaptopurine, 5-fluorouracil, chloramphenicol, phenytoin, and ethionamide - have also been associated with pellagra. Malnutrition and intestinal malabsorption have been proposed as mechanisms for niacin deficiency and pellagra. ${ }^{5}$ Pellagra itself can perpetuate further malnutrition due to GI effects. ${ }^{6-7}$ Azathioprine treatment has also been reported to induce pellagra. ${ }^{8}$

There are currently no tests or laboratory markers of diagnostic value in pellagra. Abnormal 5-OH-indoleacetic acid levels in a 24-hour urine test have been proposed as a potential marker for pellagra. Elevated levels may be due to a carcinoid tumor and reduced levels could indicate tryptophan sequestration for the synthesis of niacin, causing depleted serotonin levels. ${ }^{9}$ However, whether it has any value in diagnosing is still controversial. Blood levels of NAD and NADP have not been found to correlate well with tissue levels in patients with pellagra as they are not reduced. ${ }^{5}$ The levels of the niacinamide metabolites 1-methylnicotinamide and 1-methyl-2-pyridone-5-carboxymide in relation to blood creatinine levels in an Angolan population with and at risk of pellagra was proved to be useful for identifying vulnerable populations in developing countries.

Pellagra diagnosis is generally based on skin manifestations, clinical history, and physical examination. It is characterized by the classic clinical triad of dermatitis, diarrhea, and dementia and has become rare in developed countries. However, if left untreated, a fourth " $\mathrm{D}$ ", death, may occur. Skin changes usually appear on areas exposed to the sun, heat, and pressure. The diagnosis of pellagra may be missed when a clinician looks for malnutrition only in those patients who appear undernourished. In the present case, the patient looked well nourished and with a normal weight. She showed no neurological symptoms. But we had little difficulty in diagnosing her, and the outcome of the treatment confirmed it. The histologic features of pellagra vary throughout the course of the disease and 
are nonspecific in many cases..$^{9}$ Histologically, a perivascular lymphocytic infiltrate in the upper dermis and edema in the papillary dermis can be found in the acute stages. Later on, hyperkeratosis, parakeratosis, and epidermal atrophy are observed.

Pellagra is caused by a deficiency of the coenzymes nicotinamide adenine dinucleotide (NAD) and NAD phosphate (NADP), which participate in numerous processes including glycolysis, the metabolism of amino acids and proteins, and the generation of high-energy phosphate bonds. This would explain why pellagra

\section{REFERENCES}

1. Thornton AM, Drummond CJ. An unexpected case of pellagra. Med J Aust. 2014;200:546-8.

2. Lifshitz AY, Stern F, Kaplan B, Sofer E, Sela BA, Schewach-Millet M. Pellagra complicating Crohn's disease. J Am Acad Dermatol. 1992;27:620.

3. Zaraa I, Belghith I, El Euch D, Karoui S, Mokni M, Fillali A, et al. A case of pellagra associated with megaduodenum in a young woman. Nutr Clin Pract. 2013;28:218-22.

4. Shibata K, Fukuwatari T. The metabolites in the tryptophan degradation pathway might be useful to determine the tolerable upper intake level of tryptophan intake in rats. J Nutr. 2012;142:2227S-2230S.

5. Creeke PI, Dibari F, Cheung E, van den Briel T, Kyroussis E, Seal AJ. Whole blood NAD and NADP concentrations are not depressed in subjects with clinical pellagra. J Nutr. 2007;137:2013-7.

6. Brown TM. Pellagra: an old enemy of timeless importance. Psychosomatics. 2010;51:93-7 mainly affects tissues with a high rate of cell turnover, such as the skin and the digestive tract, and tissues with high energy needs, such as the brain. Niacin (or its amide niacinamide) is absorbed directly through the intestinal wall or synthesized from the essential amino acid tryptophan. ${ }^{910}$ Most IBD patients have dysfunction in absorption, and strict diet needs might put them to a dilemma.

In conclusion, since pellagra still occurs, we should take this disease into account in cases of CD patients with strict diet needs, especially with the predisposing factor of poor diet and serious diarrhea, and for those treated with immunomodulators. Pellagra can be an easily treated condition if timely recognized.
7. Varella Morandi Junqueira-Franco M, Ernesto Troncon L, Garcia Chiarello $P$ do Rosário Del Lama Unamuno M, Afonso Jordao A, Vannucchi H. Intestinal permeability and oxidative stress in patients with alcoholic pellagra. Clin Nutr. 2006;25:977-83.

8. Oliveira A, Sanches M, Selores M. Azathioprine-induced pellagra. J Dermatol. 2011;38:1035-7.

9. Piqué-Duran E, Pérez-Cejudo JA, Cameselle D, Palacios-Llopis S, García-Vázquez 0. Pellagra: a clinical, histopathological, and epidemiological study of 7 cases. Actas Dermosifiliogr. 2012;103:51-8.

10. Kumar JS, Subramanian VS, Kapadia R, Kashyap ML, Said HM. Mammalian colonocytes possess a carrier-mediated mechanism for uptake of vitamin B3 (niacin): studies utilizing human and mouse colonic preparations. Am J Physio Gastrointest Liver Physiol. 2013;305:G207-13.

\author{
MAILING ADDRESS: \\ Shi Hui \\ No.305, Zhongshandong $R d$, \\ Nanjing City, \\ 210002 JiangSu Province, China. \\ E-mail:32767572@qq.com
}

How to cite this article: Shi H, Wang S, Wang F, Wang Z, Lu H, Li M. Pellagra affecting a patient with Crohn's disease. An Bras Dermatol. 2017;92(6): 879-81. 